\title{
Turismo rural y comunalidad: impactos socioterritoriales en San Juan Atzingo, México*
}

\author{
Carlos Pérez-Ramírez** \\ Lilia Zizumbo-Villarreal*** \\ Recibido: 2013-08-27 Aprobado: 2013-10-15 Disponible en línea: 2014-05-30 \\ doi:I0.III44/Javeriana.CDRII-73.trci
}

Cómo citar este artículo: Pérez-Ramírez, C. y Zizumbo-Villarreal, L. (2014). Turismo rural y comunalidad: impactos socioterritoriales en San Juan Atzingo, México. Cuadernos de desarrollo rural, II(73), 17-38. doi:10.1II44/ Javeriana.CDR $\mathrm{II}-73$.trci

\section{Resumen}

El desarrollo del turismo rural en San Juan Atzingo, estado de México, ha sido impulsado por parte de diversos organismos internacionales, dependencias del Gobierno Federal, empresas del sector privado e incluso algunas organizaciones de la sociedad civil. No obstante, la actividad no se ha consolidado y está generando resultados contradictorios sobre los componentes que determinan el modo de vida y producción campesina. El trabajo identifica los impactos socioterritoriales del proyecto Ecoturismo Comunitario Tlahuica (ECT), y utiliza la propuesta de la comunalidad como marco metodológico para el análisis de las implicaciones sobre el territorio, los recursos, el debilitamiento de las estructuras organizativas, la dinámica del trabajo, reciprocidad, e incluso la transformación de los elementos culturales propios.

\section{Palabras clave autores:}

turismo; medio rural; intervención; áreas naturales protegidas; impacto; comunalidad

\section{Palabras clave descriptores:}

turismo rural; San Juan Atzingo (Mexico); condiciones rurales; áreas protegidas; impacto ambiental; recursos naturales comunales; comunidades rurales

*El presente trabajo es resultado de la investigación Intervención e impactos del turismo rural en áreas naturales protegidas. Análisis desde la perspectiva comunalitaria, del programa de Doctorado en Ciencias Ambientales de la Universidad Autónoma del estado de México, desarrollada con el apoyo del Consejo Nacional de Ciencia y Tecnología (Conacyt-México).

**Doctor en Ciencias Ambientales. Universidad Autónoma del estado de México, Facultad de Planeación Urbana y Regional. Correo electrónico: caperezr@uaemex.mx

*** Doctora en Sociología. Universidad Autónoma del estado de México, Facultad de Turismo y Gastronomía. Correo electrónico: lzvo4@yahoo.com 


\title{
Rural Tourism and Commonality: Socio-territorial Impacts in San Juan Atzingo, Mexico
}

\begin{abstract}
The development of rural tourism in San Juan Atzingo, state of Mexico, has been promoted by diverse international organizations, federal government agencies, private sector companies and even some civil society organizations. However, the activity has not been established and it is generating contradictory results on the components that determine the peasants' lifestyle and production. The paper identifies the socio-territorial impacts of the project Tlahuica Community-based Ecotourism (ECT), and it uses the commonality proposal as a methodological framework for analyzing the implications for the territory, the resources, the weakening of the organizational structures, the dynamics of labor, reciprocity, and even the transformation of typical cultural elements.
\end{abstract}

Keywords authors:

tourism; rural; intervention; protected natural areas; impact; commonality

Keywords plus:

rural tourism; San Juan Atzingo (Mexico)- rural conditions; protected areas; environmental impact; natural resources; rural communities

\section{Tourisme rural et communalité: les impacts socio-territoriaux à San Juan Atzingo, Mexique}

\section{Résumé}

Le développement du tourisme rural à San Juan Atzingo, un état de Mexique, a été stimulé par de différentes agences internationales, organisations gouvernementales fédérales, entreprises privées et même quelques organisations de la société civile. Néanmoins, l'activité ne s'est pas consolidée et est en train d'entraîner des résultats contradictoires sur les composants qui déterminent la manière de vivre et la production paysanne. Le travail identifie les impacts socio-territoriaux du projet Écotourisme Communautaire Tlahuica (ECT) (Ecoturismo Comunitario Tlabuica - ECT), qui se sert de la proposition de la communauté en la faisant son cadre méthodologique pour analyser des implications sur le territoire, les ressources, l'affaiblissement des structures organisationnelles, la dynamique du travail, la réciprocité, et même pour analyser la transformation des éléments culturels propres.

\section{Mots-clés auteurs:}

tourisme; milieu rural; intervention; zones naturelles protégées; impact; communalité

\section{Mots-clés descripteur:}

tourism rural; San Juan Atzingo (Mexico); conditions rurales; espaces protégés; impact sur l'environnement; ressources naturelles; communautés rurales 


\section{Introducción}

Desde la década de los años setenta, la actividad turística se ha proyectado como una estrategia fundamental para el crecimiento económico de los países en desarrollo, por lo que diversos organismos internacionales han impulsado su diversificación y consolidación. En América Latina, el Banco Mundial (BM), el Banco Interamericano de Desarrollo (BID), la Organización Internacional del Trabajo (OIT), la Organización de las Naciones Unidas para la Educación, la Ciencia y la Cultura (Unesco), la Organización para la Cooperación y el Desarrollo Económico (OCDE), y la Unión Internacional de Organismos Oficiales de Turismo (Uioot) (actualmente Organización Mundial del Turismo), han contribuido al crecimiento del sector.

Esto ha sido posible al asumir una función estratégica en los procesos de planificación de la actividad, mediante la identificación y valoración de la vocación recreativa de los recursos, la creación de políticas públicas e instituciones específicas que atendieran las demandas del sector, el financiamiento de infraestructura, equipamiento y empresas turísticas, la capacitación de prestadores de servicios, e incluso, el análisis de las tendencias y los mecanismos de comercialización de la actividad (World Bank, 1972).

En esta dinámica de asistencia internacional para el desarrollo de productos turísticos específicos, se delinearon e instrumentaron acciones que favorecieron la transformación o creación de nuevos destinos de litoral, como enclaves económicos para el desarrollo regional. De esta manera, se impulsaron los destinos de sol y playa de mayor trascendencia para el turismo internacional en México. No obstante, en la actualidad se ha emprendido la diversificación de la actividad como estrategia para intensificar su impacto económico y social, a escala regional o local y con énfasis en los grupos vulnerables, lo que incluye su intervención en las Áreas Naturales Protegidas (ANP), como una de las pocas actividades económicas permisibles en su interior (Altés, 2006; 2008).

Así, la asistencia internacional para el desarrollo turístico se ha extendido a otros espacios de interés colonial, histórico, arqueológico y, en particular, al medio rural, donde la inclusión de los bienes naturales y culturales de las comunidades campesinas al mercado turístico, genera numerosos desplazamientos de personas, pero también profusas transformaciones socioterritoriales, a partir de la transición de un modelo de desarrollo basado en las prácticas agropecuarias tradicionales, a otro fundamentado en la inserción en la economía internacional por medio del turismo (Blázquez y Cañada, 20II). 
Esta conversión económica es soportada por los argumentos del desarrollo social inducido, como una serie de intervenciones premeditadas cuya finalidad es acelerar y dirigir el crecimiento económico y el desarrollo social (Cernea, 1995). De esta manera, diversos actores externos pueden inducir cambios positivos en las comunidades campesinas, mediante su incorporación a la prestación de servicios turísticos, como la generación de empleos que eventualmente puede atenuar su inclusión en los procesos migratorios con fines laborales. Sin embargo, algunas intervenciones inadecuadas también pueden acelerar la transformación de los valores y prácticas políticas, económicas, socioculturales y con el entorno, que han definido históricamente a las colectividades rurales; por esto, resulta ineludible comprender los impactos que el turismo asistido puede generar en detrimento de las dinámicas y la forma de vida comunitaria.

Tal es el caso de la comunidad campesina de San Juan Atzingo, ubicada en el municipio de Ocuilan en el estado de México, donde una parte de su territorio fue incorporado desde 1936 al ANP Parque Nacional Lagunas de Zempoala (PNLZ), aunque persiste el régimen de tenencia comunal de la tierra. En la actualidad, múltiples actores, desde organismos internacionales, dependencias del Gobierno Federal e incluso algunas organizaciones de la sociedad civil, asisten el desarrollo la actividad turística en el predio denominado Laguna Tonatiahua, con el fin de impulsar la conservación ambiental y el bienestar social. No obstante, la realidad dista del escenario proyectado, debido a la débil afirmación de las iniciativas formuladas y las contradicciones que ha propiciado.

El presente trabajo tuvo como principal objetivo identificar los impactos socioterritoriales que el turismo rural está generando en San Juan Atzingo, con base en la perspectiva analítica de la comunalidad y la valoración de los elementos que la forman: territorio y recursos, organización comunitaria, trabajo mutuo y reciprocidad, así como los elementos culturales propios. El artículo retoma como marco metodológico los planteamientos de Pérez-Ramírez, Zizumbo-Villarreal, Monterroso-Salvatierra y Madrigal-Uribe (20I2), que pretende aportar, desde las ciencias ambientales, un modelo interpretativo sustentado en los componentes que determinan el modo de vida y la producción campesina para el análisis del desarrollo de la actividad y su impacto en la dinámica comunitaria.

\section{San Juan Atzingo y el proyecto turístico en el PNLZ} San Juan Atzingo es una comunidad campesina de origen indígena, ubicada en el municipio de Ocuilan, estado de México. Se localiza a 2560 metros sobre el 
nivel del mar (msnm); se destaca el cerro de Zempoala como la mayor elevación a $3500 \mathrm{msnm}$. Su extensión territorial es cercana a 25000 hectáreas de propiedad comunal, conforme a una resolución de 1952, por parte la Secretaría de la Reforma Agraria (SRA); de estas, 15000 hectáreas se caracterizan por la presencia de bosque de coníferas con especies de pino, oyamel, cedro, aile, encino laurelillo, encino ahuatepoxtle, palo amarillo, aguacatillo y fresno. Cuenta con abundantes recursos hídricos que favorecen a la localidad, como el río Chalma y diversos manantiales como Chorrito, Las Canoas, El Carrizo, Las Chias, El Aguacate, Tepetongo, Las Tripitas, San Antonio y Puxanda (Castaños, 2006).

Estas características físicas de la región fueron el argumento central para la creación del ANP Parque Nacional Lagunas de Zempoala (PNLZ) en 1936, con modificación de sus límites en 1947. El propósito de su creación fue asegurar la conservación de los bosques de coníferas, evitar los perjuicios de la erosión, la modificación y agotamiento de los cuerpos de agua y sus vertientes, garantizar espacios adecuados para el recreo popular, entre otros (Presidencia de la República, 1936). Actualmente el PNLZ tiene una superficie total de 4790 hectáreas, abarca los municipios de Huitzilac en el estado de Morelos y Ocuilan en el estado de México, en particular, el predio denominado Lago de Tonatiahua de la comunidad de San Juan Atzingo. Se calcula que recibe 150000 visitantes al año, por lo que la prestación de servicios turísticos constituye la actividad productiva más importante (Comisión Nacional de Áreas Naturales Protegidas [Conanp], 2008; ECT, 2007).

San Juan Atzingo se distribuye en 17 delegaciones y su población asciende a 949 personas, de los cuales solo 2I8 aún se comunican en la lengua tradicional (Tlahuica) y representa el grupo con menor número de hablantes de lengua indígena (HLI) en el estado de México (Instituto Nacional de Estadística y Geografía [Inegi], 20ro; Castaños, 2006). Esto no implica necesariamente la pérdida de su identidad y lógica comunitaria, pues esta se afirma en otros importantes componentes socioterritoriales que les han permitido dar continuidad a sus estructuras políticas, económicas y sociales.

En la comunidad persiste un sistema tradicional de gobierno, que ha posibilitado la construcción de "un proyecto político de identidad étnica en un contexto de comunidades mestizas” (Álvarez, 2006, p. 30). El sistema se soporta en la dualidad de la jerarquía cívica y religiosa, mediante la rotación cíclica permanente de cargos para la toma de decisiones. En este esquema, la población joven debe cumplir previamente con las tareas y funciones asignadas en niveles inferiores (ventenas, comandantes/ semanero, brazos), con el fin de avanzar de forma paulatina en una escalera graduada de cargos de mayor responsabilidad y poder (comisariados, delegados/mayordomos, 
mandones). De este modo se permitiría que las estructuras políticas de mayor incidencia para la toma de decisiones sean, de modo eventual, integradas por aquellos que han cumplido con propiedad funciones asignadas por la comunidad.

La población registra un alto grado de marginación con -0.535415986 (Consejo Nacional de Población [Conapo], 20I0), que indica la difícil situación económica y social que enfrentan sus habitantes, y que limita sus posibilidades de desarrollo social, si se consideran los índices de educación, vivienda, cobertura de servicios públicos y la disponibilidad de bienes. De esta forma, aunque existe un reducido porcentaje de población analfabeta (9.5\%), 32.22\% no cuentan con estudios terminados de nivel básico; la población carece de instituciones educativas y tiene que desplazarse hasta la cabecera municipal (el municipio de Tianguistenco e incluso a las ciudades de Toluca y Cuernavaca Morelos) para acceder a una formación de nivel superior. Además, aunque se registra un elevado porcentaje en la cobertura de servicios públicos en las I35 viviendas de la localidad, 5.3\% no cuenta con agua potable, $3.73 \%$ carece de energía eléctrica y solo 8.21\% no cuenta con drenaje ni excusado, mientras que el nivel hacinamiento supera 52.24\%.

Asimismo, se registra un elevado porcentaje de viviendas con piso de tierra (32.84\%), que expone a la población local a potenciales enfermedades e infecciones respiratorias y cutáneas. Sobre la disponibilidad de bienes destaca que más de $94 \%$ carece de una computadora, $43 \%$ no dispone de lavadora y $53 \%$ no cuenta con un refrigerador, en adición a esto con $82.2 \%$ que carece de una televisión (Inegi, 2010).

Las actividades vinculadas con el sector agropecuario han constituido el ingreso económico tradicional de la población, entre las cuales se destaca el cultivo de maíz y fríjol, llevado a cabo de temporal y con bajos rendimientos, aunque también se han introducido cultivos comerciales como haba, zanahoria, chícharo y papa, debido a la presión que ejerce el mercado y las poblaciones mestizas que la rodean (Álvarez, 2005). La producción se destina, por lo general, al consumo doméstico, aunque en algunos casos son comercializados en el mercado local e incluso a escala regional.

Resalta también el aprovechamiento forestal de las tierras comunales, que ha permitido la obtención de insumos para la construcción de sus viviendas, la elaboración del tejamanil e incluso la preparación de alimentos. Incluso, el trabajo de protección del bosque de la comunidad de San Juan Atzingo fue reconocido con la mención honorífica del Premio Nacional al Mérito Ecológico 2007, que otorga la Secretaría del Medio Ambiente y Recursos Naturales (Semarnat); no obstante, en la actualidad persisten diversos conflictos para el desarrollo de esta actividad, a raíz de la disputa por la posesión legal del territorio, la invasión de terrenos y la tala 
clandestina de la cubierta forestal. Además, una parte de su población se dedica a la crianza de aves de corral y de ganado menor, que satisface sus necesidades básicas (Consejo Estatal para el Desarrollo Integral de los Pueblos Indígenas del Estado de México [Cedipiem], 2009).

Se calcula que cerca de $9 \%$ de la población participa en el proceso migratorio de carácter temporal con fines laborales, en actividades como la construcción o el trabajo doméstico en las ciudades de Toluca y México D.F., y también en Canadá y Estados Unidos, principalmente en las ciudades de Sacramento, San José, Los Ángeles, Carolina del Norte y Washington (Castaños, 2006). Asimismo, los habitantes se han incorporado al trabajo asalariado y la oferta de actividades recreativas y de alimentos en el PNLZ (García, 1999), para atender las corrientes de turistas y visitantes provenientes del estado de Morelos, Distrito Federal y el propio estado de México.

Si bien el desarrollo del turismo en el parque es resultado de un prolongado proceso, donde han participado de manera espontánea las poblaciones locales, en los últimos años se han intensificado las acciones provenientes del exterior para la afirmación y diversificación de la actividad, con el argumento de impulsar la conservación ambiental. Así, múltiples organismos internacionales, dependencias del gobierno federal, estatal y municipal e incluso organizaciones de la sociedad civil impulsan hoy la actividad turística, no solo en el área recreativa del PNLZ sino también en predios colindantes como laguna Tonatiahua, localizada al pie del cerro de Las Alumbres y que pertenece a San Juan Atzingo.

El BID, como organismo ejecutor del Fondo para el Medio Ambiente Mundial (FMAM) o The Global Environment Facility (GEF), ha canalizado recursos por intermedio del Fondo Mexicano para la Conservación de la Naturaleza AC (FMCN) para el financiamiento de diversos programas de conservación y biodiversidad, que incluyen el Fondo para las Áreas Naturales Protegidas (FANP), y el Sistema Nacional de Áreas Naturales Protegidas (Sinap). Este último ha apoyado a distintas ANP en los rubros de Gasto Corriente y Proyectos Innovadores Estratégicos de Conservación (Rickards y Piguerón, 2003; FMCN, 2010), entre estos, la prestación de servicios turísticos. En el año 2002 se otorgaron recursos económicos por USD\$I 800000 , para dar respuesta a necesidades financieras, de equipo y de personal del Corredor Biológico Chichinautzin (Cobio), que comprende un Área de Protección de Flora y Fauna y dos parques nacionales (lagunas de Zempoala y El Tepozteco) (Parkswatch, 2002; Pérez-Ramírez, Zizumbo-Villarreal, Romero-Contreras, Cruz-Jiménez, y Madrigal-Uribe, 20II). 
Del mismo modo, el Gobierno Federal mediante la Conanp y la Comisión Nacional para el Desarrollo de los Pueblos Indígenas (CDI), al reconocer la importancia de la diversidad de flora y fauna silvestre de la región y, en especial, los servicios ambientales que brinda (como el suministro de agua potable para los núcleos urbanos de la Ciudad de México y los estados de México y Morelos), han contribuido al desarrollo de la actividad turística como estrategia para su conservación, mediante una inversión aproximada de 3.8 millones de pesos mexicanos en el Proyecto Ecoturístico Comunitario Pueblo Tlahuica San Juan Atzingo (ECT) de la laguna Tonatiahua. Esta iniciativa proyectó la construcción de un área de acampado, miradores, senderos interpretativos, oferta de actividades recreativas, elaboración de una guía de interpretación ambiental y trípticos alusivos al proyecto, lo que beneficia de manera directa a 96 personas (Conanp, 2007).

Por su parte organizaciones de la sociedad civil y la iniciativa privada también han intervenido en el desarrollo de la actividad como estrategia para la conservación. En 2008 Naturalia AC inició un proyecto de reforestación de 20000 árboles en la región, y amplió sus acciones a la promoción del turismo como alternativa económica para la población. Para ello, en 2009, con el respaldo de la institución bancaria HSBC y una inversión de I.7 millones de pesos mexicanos, impulsaron el programa Comunidades Sustentables: San Juan Atzingo-Zempoala. Este tuvo como objetivo consolidar diversos proyectos de conservación y restauración de los recursos naturales, así como proyectos productivos, educativos y de capacitación para el mejoramiento de las condiciones de vida de la población.

La iniciativa establecía, para un plazo de cinco años, la instalación de un vivero de producción forestal, la prestación de servicios turísticos, la creación de cooperativas para la venta de artesanías y miel de maguey, cursos de educación ambiental en escuelas, e incluso la proyección de un corredor ecoturístico a escala regional, dirigido las corrientes de visitantes extranjeros y nacionales (Naturalia, 2009; ECT, 2007; 2009; HSBC, 2010; Pérez-Ramírez, et ál., 20II).

Otros organismos internacionales, dependencias públicas y organizaciones sociales han respaldado la iniciativa, como el Banco Mundial, Green World Campaign, Semarnat, Secretaría de Turismo (Sectur), Comisión Nacional Forestal (Conafor), Procuraduría Federal de Protección al Ambiente (Profepa) y Protectora de Bosques del Estado de México (Probosque).

Sin embargo, a pesar de que el proyecto de turismo rural en Tonatiahua ha sido respaldo por múltiples actores, e incluso recibió la anuencia de las entonces autoridades locales (representadas por el Comisariado de Bienes Comunales), en la 
actualidad se revela como una iniciativa indefinida e inoperante, donde son pocos los habitantes que se han interesado verdaderamente en el proyecto. Además, la infraestructura y el equipamiento instalado para la prestación de servicios turísticos han sido destruidos, abandonados y se deterioran cada vez más con el paso del tiempo, debido a las condiciones climáticas y su inapropiada utilización.

Este escenario refleja el limitado alcance mecanismos de intervención, centrados en la adecuación del espacio por encima de la dimensión social del desarrollo. Frente a esta realidad, es necesario comprender cuáles son los impactos socioterritoriales que el desarrollo turístico genera en San Juan Atzingo, a partir de la comunalidad como marco para el análisis de las implicaciones sobre el territorio y los recursos, el debilitamiento de las estructuras organizativas, la dinámica del trabajo, la reciprocidad y la transformación de los elementos culturales propios.

\section{Metodología: perspectiva de análisis desde la comunalidad}

Para el estudio de los impactos socioterritoriales que ha generado el impulso a la actividad turística en San Juan Atzingo, se retoma como eje de análisis la propuesta de la comunalidad. Esta constituye una noción en construcción, cuyos planteamientos iniciales se trazaron hace casi tres décadas por campesinos, indígenas e intelectuales oaxaqueños, aunque es posible identificar múltiples perspectivas desde las que se ha abordado.

Se configura como una perspectiva ideológica que pretende reivindicar el papel de las comunidades indígenas en el proceso de desarrollo, basado en la revaloración, fortalecimiento y movilización de los elementos que les han permitido dar continuidad a su colectividad a lo largo del proceso histórico. Por tanto constituye un proceso de "desarrollo comunalitario [que] emerge como resultado de un duelo de fuerzas y de pensamientos: la imposición de modelos ajenos y la resistencia con modelos propios de pensamiento y organización” (Martínez, 2003, p. 46). Esto es, como un renovado argumento y aspiración de reconstrucción de dinámicas comunitarias, que les permita incidir efectivamente en sus carencias, limitaciones y retos actuales (Pérez-Ramírez, et ál., 20I2).

Por otra parte, según Díaz (2003, p. 367), constituye la esquematización de las dinámicas comunitarias internas, que "definen la inmanencia de la comunidad en la medida que [...] definen otros conceptos fundamentales para entender una realidad indígena", con base en la existencia de elementos fundamentales (territorio comunal, trabajo colectivo, poder político, fiesta comunal) y complementarios 
(derecho indígena, educación tradicional, lengua habitual, cosmovisión) definidos por Rendón (2003). Se trata de una configuración atomizada que distingue la importancia de los elementos centrales que dan continuidad a la lógica comunal, aun cuando los elementos auxiliares o complementarios sean alterados o asimilados por la racionalidad económica (Maldonado, 2002).

Por ello, esta noción no es una cualidad exclusiva de los pueblos indígenas, en tanto que numerosas comunidades campesinas mantienen vigentes las estructuras sociopolíticas para la toma de decisiones, la propiedad colectiva del territorio y los recursos, las relaciones de reciprocidad y participación social en el trabajo, cargos, asambleas, fiestas, así como los valores y manifestaciones culturales que afirman sus rasgos comunitarios (Maldonado, 2003). Por tanto, es posible percibir a los campesinos como sujetos sociales de la comunalidad, con la intención de indicar una relación estructural y no un contenido cultural particular, respecto del aprovechamiento recreativo de sus recursos y la prestación de servicios turísticos en el medio rural (Romero y Ávila, 200I; Pérez-Ramírez, et ál., 2012).

De esta manera, la comunalidad se revela en dos contextos paralelos y suplementarios, como aspiración de un proceso de desarrollo incluyente, tanto como en cuanto al diseño atomizado de los elementos fundamentales y complementarios que soportan la lógica comunal. Sin embargo, en el entramado de estas posiciones, es posible identificar una perspectiva analítica sobre las dinámicas campesinas y su vínculo con las interacciones políticas, económicas y socioculturales que transforman el entorno rural.

En este sentido, la comunalidad puede ser asumida como "una forma de nombrar y entender el colectivismo" (Maldonado, 2002, p. 72), dado que constituye una alusión a sus dinámicas, pero también una forma en la cual los no indígenas pueden observarlos; por este motivo, su estudio debe partir desde una perspectiva analítica que permita comprender los elementos inherentes a la comunalidad. De acuerdo con esto, Korsbaek (2009) destaca la comunalidad en la búsqueda y construcción de un marco explicativo acerca de los deseos y necesidades de la población campesina, pero también reconoce que constituye un enfoque interno a partir del cual se puede definir un proyecto propio.

El trabajo se sitúa en los planteamientos de Pérez-Ramírez (et ál., 20I2), sobre la comunalidad como marco para el estudio de dinamización turística en el espacio rural que, desde las ciencias ambientales, pretende aportar una perspectiva distinta de los procesos socioterritoriales que intervienen en la afirmación de la actividad en las comunidades campesinas. El estudio se basa en los siguientes criterios de análisis: 
a. Territorio y recursos de aprovechamiento turístico: con estos elementos se ha construido una serie de mecanismos sociales para la organización sociopolítica, que incide tanto en la apropiación colectiva de los recursos como en su aprovechamiento con fines turísticos. Además, es justamente el territorio el que puede afianzar, a partir del turismo, las condiciones de arraigo, cohesión social, reciprocidad y la formación de proyecto común para la solución a problemáticas compartidas.

b. Organización comunitaria en la prestación de servicios: en los procesos de participación colectiva y toma de decisiones de las comunidades campesinas, destaca la importancia de la estructura cívica y religiosa, cuya fortaleza e integridad permite afianzar la cohesión social y la autogestión entre la población. De este modo se incide de manera directa en la planeación, desarrollo y ejecución del turismo; pero, en el caso contrario, el desgaste y fragmentación de este sistema indica la penetración del mercado en su estructura, lo que favorece la división del interés comunitario para afirmar logros individuales.

El estudio se basa en los siguientes criterios de análisis:

c. Territorio y recursos de aprovechamiento turístico: con estos elementos se ha construido una serie de mecanismos sociales para la organización sociopolítica, que incide tanto en la apropiación colectiva de los recursos como en su aprovechamiento con fines turísticos. Además, es justamente el territorio el que puede afianzar, a partir del turismo, las condiciones de arraigo, cohesión social, reciprocidad y la formación de proyecto común para la solución a problemáticas compartidas.

d. Organización comunitaria en la prestación de servicios: en los procesos de participación colectiva y toma de decisiones de las comunidades campesinas, destaca la importancia de la estructura cívica y religiosa, cuya fortaleza e integridad permite afianzar la cohesión social y la autogestión entre la población. De este modo se incide de manera directa en la planeación, desarrollo y ejecución del turismo; pero, en el caso contrario, el desgaste y fragmentación de este sistema indica la penetración del mercado en su estructura, lo que favorece la división del interés comunitario para afirmar logros individuales.

e. Trabajo mutuo y reciprocidad para el turismo rural: se presenta en diversos niveles de articulación social al interior y exterior de las propias comunidades, por tanto, se deben considerar las relaciones existentes desde la unidad doméstica, la comunidad como manifestación de intereses colectivos y las interacciones intercomunitarias en lo regional; al mismo tiempo, deberán considerarse las redes de reciprocidad, que permiten la reproducción de ayuda mutua, así como la construcción lazos sociales de colaboración, emanados del servicio voluntario al 
otro, que lo vincula y obliga a la correspondencia, y también propicia la integración y convivencia entre los actores sociales.

f. Elementos culturales propios y su configuración en el turismo: constituyen características socioculturales que definen a una colectividad, por lo que es necesario identificar la permanencia, transformación o pérdida de elementos de cohesión social como fiestas, ritos, creencias, cosmovisión, lengua, música, manifestaciones artísticas, vestido, alimentación y la forma como inciden en el aprovechamiento turístico de los recursos. Su pérdida o asimilación paulatina al mercado, sin embargo, no constituye necesariamente una limitación para la comunalidad, pues aunque algunos elementos hayan desaparecido o se hayan transformado, puede persistir en los tres componentes fundamentales anteriores (Pérez-Ramírez, et ál., 20I2).

Con base en estos planteamientos, se diseñaron, probaron y aplicaron distintos instrumentos de investigación de campo, como un guión semiestructurado y flexible de entrevista, aplicado a representantes locales que integran los comisariados de Bienes Ejidales y Comunales, comités de Vigilancia, delegados municipales, prestadores de servicios turísticos, profesionales, amas de casa y adultos mayores (lo que permitió profundizar en los impactos del turismo en el lugar de estudio mediante el análisis del discurso). Además, destaca el diseño, prueba y aplicación de una Lista de Verificación de Impacto Ambiental (LVIA) y una Matriz Cruzada de Impacto Ambiental (MCIA), que permitieron la identificación y valoración de los impactos físicos que ha generado el turismo.

\section{Resultados}

\section{I. Territorio y recursos de aprovechamiento turístico}

La diversidad biológica y profusión de los recursos forestales e hídricos de San Juan Atzingo ha sido objeto de permanentes disputas a lo largo del proceso histórico. Esto, pese a que sus propias estructuras sociopolíticas, definidas por el sistema comunal de posesión de la tierra, le han permitido soportar el ahínco externo por fragmentar y explotar sus recursos. Así, la institucionalidad agraria constituye el mecanismo central para el reconocimiento y afirmación del territorio y los recursos de uso común, que aparentemente facilita el usufructo proporcional de la población.

Si bien con la creación del ANP en 1936, una parte de su territorio quedó comprendida en los límites del PNLZ, la comunidad siempre ha ejercido sus derechos 
sobre la tierras y los recursos bajo el régimen de tenencia comunal, dado que el decreto asumió un carácter enunciativo y no expropiatorio (Conanp, 2008). Sin embargo, esa categorización incidió en el manejo de los recursos naturales, al regular el desarrollo de actividades económicas tradicionales, como el aprovechamiento forestal, e impulsar nuevas iniciativas productivas como el turismo rural en la laguna Tonatiahua.

Es claro que el turismo tiene su propia dinámica e impactos sobre el medio físico, aunque el limitado desarrollo de la actividad en San Juan Atzingo, debido al abandono del proyecto y la exigua presencia de usuarios, no ha generado impactos significativos. Pese a esto, derivado de la aplicación de la LVIA y la MCIA, es posible identificar determinadas alteraciones sobre el medio físico y biótico, que generó la adecuación del espacio para la construcción de infraestructura y equipamiento turístico, en particular, con la delimitación del camino de acceso, senderos, sanitarios, puentes, miradores, área de juegos infantiles, palapas, comedores y zona de acampado. Además, la presencia eventual de algunos visitantes en la laguna Tonatiahua y la realización espontánea de actividades recreativas como senderismo, bicicleta de montaña (MTB), motocross, cabalgatas, campismo, fogatas y preparación de alimentos, ocasionaron determinados impactos físicos dada la contaminación con residuos sólidos, la compactación y erosión del suelo, destrucción de la cubierta vegetal, perturbación de especies y alteración del paisaje natural.

Aunque se reconoce que el turismo rural no presenta una amenaza para la fragmentación del territorio (en tanto que el predio donde se proyectó la iniciativa no puede ser vendido u otorgado bajo cesión a particulares, y tampoco se han configurado formas disimuladas de propiedad privada que puedan ser heredadas, cedidas e incluso comercializadas en el interior del núcleo agrario), es posible que puedan formarse mecanismos de regulación y participación abierta que permitan la intervención en el manejo de los recursos en el ANP.

No obstante, más allá de las iniciativas turísticas y las implicaciones ambientales que genera para la comunidad, existen otras importantes amenazas que enfrentan las localidades de la región, no solo para conservar la integralidad de las tierras ejidales o comunales, sino para la propia continuidad de la institucionalidad agraria y la dinámica campesina que las caracteriza. $\mathrm{Al}$ respecto, existe un pleno interés de diversos actores externos en el aprovechamiento de sus recursos, en particular, la canalización de recursos hídricos hacia las zonas urbanas, la explotación comercial y clandestina de los bosques, la incorporación de las tierras colectivas en el mercado inmobiliario, así como la construcción de la autopista Lerma-Tres Marías (LjM), que se proyecta para conectar los estados de México y Morelos. 


\subsection{Organización comunitaria en la prestación de servicios turísticos}

La puesta en marcha de proyectos de turismo en el medio rural, que precisan la incorporación de los actores locales para la oferta de servicios recreativos, transforman los esquemas de organización característicos de las comunidades campesinas, pues afirman modelos laborales distintos a los tradicionales. Si bien este proceso puede favorecer el surgimiento de nuevas estructuras y liderazgos locales enfocados en el desarrollo de la actividad, también puede generar severas implicaciones para el bienestar colectivo, en tanto debilitan la institucionalidad agraria como soporte de la lógica comunitaria.

En el caso del proyecto en Tonatiahua, a pesar de que múltiples organismos internaciones, instituciones públicas, organizaciones sociales e incluso los propios integrantes del Comisariado de Bienes Comunales respaldaron la iniciativa turística (al canalizar financiamiento y recursos económicos para la construcción de infraestructura, equipamiento y capacitación de los nuevos prestadores de servicios), tan solo algunos habitantes incursionaron en el turismo, por lo que el desarrollo de la actividad no ha incidido de forma notable en las formas de organización comunitaria.

En este sentido, el turismo no ha sido factor para que en San Juan Atzingo se constituyan nuevas instituciones enfocadas en la planificación, operación y regulación de la actividad, por lo cual, no se le puede atribuir el potencial debilitamiento de la participación social en las estructuras locales. La forma de gobierno que persiste en la comunidad se fundamenta en una jerarquía cívicoreligiosa, dirigida por los habitantes que han demostrado mayor compromiso y responsabilidad en las funciones y cargos asignados. En la actualidad las estructuras agrarias se están reconfigurando, incluso con la participación directa de jóvenes, con base en su mayor preparación académica y visión de desarrollo económico.

Pese a lo anterior, el apoyo económico y material que se recibió para impulsar el turismo contribuyó a la inestabilidad política de la colectividad, debido a la escasa información difundida respecto del manejo de los recursos y el resguardo personal de algunos equipos por parte de los representantes. Esto, sumado a otros conflictos internos por la explotación forestal, derivó en el debilitamiento del Comisariado de Bienes Comunales, el surgimiento de grupos de interés al interior de las estructuras locales y la intensificación de disputas por el ejercicio del poder y la toma de decisiones en la comunidad.

Además, si bien a lo largo del tiempo se han registrado numerosas disputas y litigios legales por la delimitación territorial, que han enfrentado a la población con las localidades contiguas en cuanto al desarrollo turístico, se han incrementado 
los conflictos en el ámbito intercomunitario, en especial con Huitzilac, por la posesión del predio Tonatiahua. Esta situación se debe a la potencial rivalidad con los prestadores de servicios instalados en el parque nacional, y la centralización de la eventual derrama económica, que ha derivado en la destrucción de la infraestructura instalada y lesiones entre la población. De esta forma se ha debilitado la correspondencia intercomunitaria y la posible integración regional en la construcción de iniciativas de mayor alcance, que den respuesta a problemáticas compartidas.

\subsection{Trabajo mutuo y reciprocidad para el turismo rural}

Con la definición de uso público de un área natural y el impulso para el aprovechamiento recreativo, no solo se generan transformaciones en el espacio físico, se adaptan los mecanismos de acceso y control de los recursos, o forman estructuras organizativas paralelas que alteran la dinámica de la institucionalidad agraria, sino que se producen cambios en los esquemas de trabajo en lo individual, en la unidad doméstica y la propia comunidad.

En el marco de la apertura de la laguna Tonatiahua para el desarrollo de actividades turísticas, no se ha intensificado el proceso de abandono y reconversión productiva de la población con respecto al desplazamiento de actividades económicas tradicionales, como el cultivo del maíz o el aprovechamiento forestal, para la incorporación en la prestación de servicios turísticos y el abastecimiento de los insumos necesarios para la oferta de actividades recreativas. Tampoco se ha acentuado la rivalidad individual por la prestación de servicios que pudiese alterar la intencionalidad del trabajo colaborativo, la reciprocidad o la ayuda mutua entre los actores locales. Del mismo modo, el escaso interés de la población por participar en el turismo ha frenado el ajuste de las dinámicas económicas y participativas de los integrantes de la unidad doméstica, para la diversificación del ingreso, mediante la oferta de productos y servicios a las corrientes de visitantes.

Sin embargo, en el ámbito comunitario fue evidente la adaptación de los esquemas organizativos con la finalidad de ser partícipes del financiamiento y canalización de recursos para el despliegue de la actividad. Esto implicó la asimilación de nuevas estructuras definidas por el mercado, como una condición ineludible para acceder a los recursos, y pretender su alineación como una entidad corporativa homogénea, capaz de hacer frente a las obligaciones contraídas.

Si bien la restringida participación de los actores locales en el turismo no ha intensificado la reconfiguración económica de San Juan Atzingo, existen otras variables socioeconómicas que han afirmado la apertura al exterior en función 
de los intereses del mercado. La mayoría de estas variables se derivan del complejo escenario de la producción y comercialización agropecuaria, la inserción en los procesos migratorios con fines laborales, la incorporación al trabajo asalariado, así como el impulso de nuevos proyectos productivos y cultivos comerciales.

\subsection{Elementos culturales propios y su configuración para el turismo}

Respecto a los impactos del turismo rural sobre los elementos de cohesión social como la fiesta, ritos, creencias, cosmovisión, lengua, música, manifestaciones artísticas, vestido y alimentación, que han afirmado históricamente a las comunidades campesinas, es claro que la actividad turística por su propia orientación y alcances, lleva implícita su transformación y adaptación, dado que la apertura al exterior favorece la asimilación de prácticas, valores y comportamientos distintos a los reproducidos de forma cotidiana en el ámbito comunitario. También es justo reconocer que, a partir del desplazamiento de las corrientes turísticas y la puesta en valor de los elementos culturales, es posible la revaloración y conservación de componentes fundamentales de identidad colectiva, y propiciar la generación de alternativas económicas para la población.

El turismo en San Juan Atzingo no ha impactado en la fiesta como soporte fundamental del sistema sociopolítico local que, más allá del carácter simbólico de las festividades cívicas o religiosa, permite la reproducción de formas de participación y mecanismos de redistribución al interior de la colectividad. La débil afirmación de la actividad limita la generación de ingresos para hacer frente a los gastos del patrocinio de alguna festividad, por lo que recurren a otras alternativas productivas que les permitan cumplir con su compromiso y ser partícipes del sistema jerárquico. Tampoco se tiene registro de nuevas configuraciones festivas como resultado de la dinamización turística en el lugar de estudio.

Del mismo modo, el exiguo desplazamiento de turistas y visitantes tiene poca relevancia respecto a la modificación o revivificación de ritos, creencias y la cosmovisión local, que se conservan en el escenario particular de la población. La lengua y el vestido tradicional son objeto de un histórico proceso de abandono que amenaza seriamente su continuidad, pero la presencia de turistas no acentúa de modo directo su detrimento ni tampoco ha constituido un argumento sólido para su revaloración. Incluso, respecto a las manifestaciones musicales, artísticas y la cultura alimentaria de la región, no tienen una presencia implícita en la iniciativa, por tanto su transfiguración es invalidada. No obstante, estas expresiones se mantienen vigentes y se reproducen en lo cotidiano con relativa vinculación hacia la actividad 
turística en el PNLZ, que ha derivado en una adaptación acorde con las necesidades y expectativas de los usuarios.

Con base en lo anterior, no existe evidencia de alteración significativa sobre los elementos culturales propios, atribuible de forma directa con el desarrollo de la actividad en Tonatiahua, en tanto que sus consecuencias no se reflejan en la permanencia, transformación o pérdida de estos valores. Inclusive, la apertura y contacto de los elementos culturales con el mercado se produce, en esencia, dentro del marco de otras actividades productivas, escenarios políticos, ámbitos religiosos y dinámicas sociales que, en efecto, han incidido en su configuración actual.

\section{Conclusiones}

Las condiciones de pobreza y marginación que caracterizan a las comunidades campesinas en México son resultado de un prolongado proceso de exclusión y contención de sus capacidades políticas, económicas, socioculturales y de relación con el entorno, lo que limita su potencialidad como actores centrales de sus propios procesos de desarrollo. Incluso el ahínco histórico del mercado, por apropiarse o participar en el manejo y explotación de sus recursos, ha derivado en la formulación e instrumentalización de diversas estrategias de intervención, que acentúan las profusas implicaciones y retos que han enfrentado a lo largo de su historia.

De esta forma, diversos actores han concebido planes, programas y proyectos como parte de los procesos de desarrollo planificado y estrategias de intervención en el medio rural, que pretenden mitigar los problemas que enfrenta la población campesina, aunque en numerosas ocasiones han aportado resultados contradictorios para el bienestar social. Persisten proyectos de intervención que desestiman la dimensión social del desarrollo, concentran los recursos en función de prioridades definidas desde el exterior, atienden unidades segmentadas de la población con atención excesiva, favorecen la formación de enclaves para el desarrollo de actividades productivas comerciales (que alteran la dinámica económica local), establecen plazos limitados de asistencia sin avalar su continuidad a futuro, e intensifican los conflictos al interior de la organización social.

Tal es el caso del proyecto de turismo rural en la laguna Tonatiahua del PNLZ, donde la población de San Juan Atzingo ha sido objeto de múltiples intervenciones que pretenden asistir la afirmación del turismo rural como estrategia para la conservación ambiental y el bienestar social. Sin embargo, tal pretensión, a pesar de contar con el respaldo de organismos internacionales, dependencias públicas 
del Gobierno Federal y organizaciones de la sociedad civil, se revela como una iniciativa endeble que ha generado diversas implicaciones políticas, económicas, socioculturales y físicas para la comunidad.

El presente trabajo tuvo como objetivo identificar los impactos socioterritoriales que la actividad genera, con base en la perspectiva analítica de la comunalidad y la valoración de los elementos que la constituyen: territorio, recursos, organización comunitaria, trabajo mutuo, reciprocidad y elementos culturales propios.

Respecto al territorio y recursos de aprovechamiento turístico, con la creación del ANP no se afectó la posesión colectiva, pues la comunidad siempre ha ejercido sus derechos sobre el territorio; sin embargo, existen múltiples intereses por su apropiación y aprovechamiento que pueden incidir en su eventual fragmentación. Además, con la intensificación de las estrategias de intervención a partir del impulso al turismo rural, se han generado determinados impactos sobre el medio físico y biótico, dada la construcción de infraestructura y equipamiento. Incluso, a pesar de la presencia espontánea de turistas en el lugar, algunas actividades recreativas que se desarrollan ocasionalmente, como senderismo, motocross, MTB, campismo y fogatas, han propiciado la contaminación con residuos sólidos, la compactación y erosión del suelo, destrucción de la cubierta vegetal, perturbación de especies y la alteración del paisaje.

En relación con la transformación de la organización comunitaria en la prestación de servicios turísticos, el incipiente desarrollo de la actividad no ha incidido de manera notable en la modificación de sus estructuras sociopolíticas, en tanto que no ha impedido la formación de nuevas instituciones que pudieran debilitar la dinámica de las estructuras cívico-religiosas. Sin embargo, a partir de la canalización de recursos destinados al despliegue de la actividad, se acentuaron los conflictos internos y la inestabilidad política del Comisariado de Bienes Comunales, además, se han intensificado los conflictos de carácter intercomunitario, debido a la potencial rivalidad y beneficios económicos que pudiese generar el proyecto en Tonatiahua.

En cuanto al trabajo mutuo y reciprocidad para el turismo rural, no se han presentado cambios significativos en los esquemas de trabajo en lo individual, trabajo colaborativo, reciprocidad o ayuda mutua atribuida directamente a la actividad. Tampoco es evidente el interés de los integrantes de la unidad doméstica por diversificar sus ingresos económicos mediante la oferta de productos y servicios a los turistas. Tan solo en el ámbito comunitario se modificaron los esquemas organizativos, con la creación de grupos de trabajo que manifestaron su interés, al inicio del proyecto, de ser partícipes en los beneficios de la actividad, y ajustarse a las exigencias y delineaciones impuestas por los actores externos. 
Finalmente, en lo que concierne a los elementos culturales propios y su configuración para el turismo, la actividad no puede ser considerada como factor determinante para la permanencia, transformación o pérdida de la fiesta, ritos, creencias, cosmovisión o lengua tradicional, pues se relacionan con dinámicas de mayor alcance que han propiciado la asimilación de prácticas, valores y comportamientos distintos a la lógica comunitaria. Estas se vinculan con el contexto político, económico, sociocultural y el aprovechamiento de los recursos de concurrencia en el ámbito regional (partidos políticos, prácticas religiosas, procesos migratorios, grado de escolaridad). Tan solo en el PNLZ se registra la adaptación de las manifestaciones musicales, artísticas y la cultura alimentaria de la región, conforme a las necesidades y expectativas de los turistas y visitantes, lo que incide efectivamente en su configuración actual.

Se retomó a la comunalidad como marco metodológico de la investigación, con la finalidad de sobrepasar un cálculo exclusivo y parcial de carácter cuantitativo, acerca de la incidencia que tiene el turismo sobre el medio físico, económico o sociocultural en San Juan Atzingo. Para ello se consideró una perspectiva de análisis de mayor alcance sobre el objeto de estudio, soportada en las ciencias ambientales, que permitió ampliar las consideraciones teóricas y metodológicas para el abordaje de la interrelación de la actividad sobre los elementos comunitarios.

Además, si bien existen determinados planteamientos para el estudio de los impactos socioterritoriales del turismo, por lo general, se han trazado como configuraciones genéricas y externas a la dinámica de las comunidades campesinas en México. En este sentido, es válido avanzar en la definición de un esquema de análisis desde abajo, fundamentado en las propias representaciones de los actores locales, en cuanto al aprovechamiento recreativo de sus recursos, la incidencia que tiene sobre el territorio, recursos, organización comunitaria, trabajo mutuo, reciprocidad y elementos culturales propios, así como su reconfiguración para la prestación de servicios.

A partir de los resultados obtenidos, se afirma que a pesar de la endeble consolidación del proyecto Ecoturismo Comunitario Tlahuica (ECT) en el Parque Nacional Lagunas de Zempoala, se están generando determinados impactos socioterritoriales sobre los elementos comunitarios de San Juan Atzingo, con un entorno físico relativamente modificado, exigua correspondencia con la reconversión productiva o la transformación del repertorio cultural, pero con mayor incidencia en los mecanismos de organización sociopolítica. Esto ha derivado en la intensificación de conflictos de carácter intrínseco y extrínseco de la comunidad.

Es preciso dar continuidad al análisis de procesos de desarrollo planificado y estrategias de intervención a partir del turismo rural, debido a que la suma de esfuerzos 
provenientes del exterior y el ineludible protagonismo de los actores locales pueden contribuir, de manera efectiva, con el mejoramiento en sus condiciones de vida y la conservación de la biodiversidad. Sin embargo, la disimulada instrumentación de acciones inicuas de intervención puede aportar al debilitamiento de la institucionalidad agraria y la lógica comunitaria, como vía para la afirmar la penetración del mercado, al utilizar el turismo como un factor más del distanciamiento en la articulación de los intereses colectivos, con potenciales efectos contradictorios para el bienestar social.

\section{Referencias}

Altés, C. (2006). El turismo en América Latina y el Caribe y la experiencia del BID. Serie de informes técnicos del Departamento de Desarrollo Sostenible, Washington D.C.: Banco Interamericano de Desarrollo. Recuperado el 30 de enero de 20II, de http://idbdocs.iadb.org/wsdocs/getdocument.aspx?docnum=I442249

Altés, C. (2008). Turismo y desarrollo en México. Nota sectorial. Banco Interamericano de Desarrollo, Departamento de Países de Centroamérica, México, Panamá y República Dominicana, Documento de Trabajo CSI-II7 ME-Pıого. Recuperado el 20 de enero de 20II, de http://cestur.sectur.gob.mx/pdf/ estudioseinvestigacion/calidadycompetitividad/publicacionesexternas/2006 BID_TurismoyDesarrolloenMexico.pdf

Álvarez, R. (2005). Etnicidad, cargos y adscripciones religiosas en dos comunidades indígenas del Estado de México. Cuicuilco, I2(034), 29-40.

Álvarez, R. (2006). Tlabuicas. Pueblos indígenas del México contemporáneo. México D. F.: Comisión Nacional para el Desarrollo de los Pueblos Indígenas.

Blázquez, M. y Cañada, E. (Eds.) (20II). Turismo placebo. Nueva colonización turística: del Mediterráneo a Mesoamérica y El Caribe. Lógicas espaciales del capital turístico. Managua: Edisa.

Castaños, C. (2006). Diagnósticos de los pueblos indígenas, grupos vulnerables, páramo del campo y la ciudad. Toluca: Centro de Estudios sobre Marginación y Pobreza.

Cernea, M. (1995). Primero la gente: variables sociológicas en el desarrollo rural. México, D. F.: Fondo de Cultura Económica.

Comisión Nacional de Áreas Protegidas [Conanp] (2007). Un nuevo ciclo de vida. Logros 2007. Recuperado el I2 de enero de 20I2, de http://centro.paot.org.mx/ documentos/conanp/logros_2007.pdf

Comité para la Conservación de Especies Silvestres, AC [Naturalia] (2009).

Desarrollo sustentable. Recuperado el ıo de mayo de 20II, de http://naturalia.mx/ naturalia/index.php/desarrollo-sustentable-I 
Conanp (2008). Anteproyecto programa de conservación y manejo Parque Nacional Lagunas de Zempoala, Corredor Biológico Chichinautzin. Dirección Regional Centro y Eje Neovolcánico. Recuperado el I2 de enero de 2009, de http://www.conanp.gob. mx/anp/consulta/Anteproyecto\%20Chichinautzin\%20y\%20mapa.pdf

Consejo Estatal para el Desarrollo Integral de los Pueblos Indígenas del Estado de México [Cedipiem] (2009). Tlabuica. Recuperado el ıo de mayo de 2010, de http://portal2.edomex.gob.mx/cedipiem/pueblosindigenas/tlahuica/index.htm Consejo Nacional de Población [Conapo] (2010). Indice de marginación por localidad. Recuperado el 20 de marzo de 2011 , de http://www.conapo.gob.mx/es/ CONAPO/Indice_de_Marginacion_por_Localidad_2010

Díaz, F. (2003). Comunidad y comunalidad. En J. Rendón, Comunalidad: modo de vida comunal entre los pueblos indios (pp. 365-373). México D. F.: Consejo Nacional para la Cultura y las Artes, Dirección General de Culturas Populares e Indígenas.

Ecoturismo Comunitario Tlahuica [ECT] (2009). Ecoturismo Comunitario Tlabuica, San Juan Atzingo, Estado de México. El proyecto: ¿Quiénes somos? Recuperado el 10 de mayo de 2010, de http://www.ecoturismotlahuica.com/ecoturismo.htm ECT (2007). Pjiekakjo. Ecoturismo Comunitario Tlabuica, San Juan Atzingo, Estado de México. Recuperado el ro de mayo de 20ro, de http://www.ecoturismotlahuica. com/ecoturismo.htm

Fondo Mexicano para la Conservación de la Naturaleza [FMCN] (20I0). Fondo para Áreas Naturales Protegidas. Manual de Operaciones. Recuperado el 2 de marzo de 20II, de http://www.conanp.gob.mx/acciones/pdf_fanp/Manual_FANP_2009.pdf García, E. (1999). Ocuilan. Monografía municipal. Toluca: Instituto Mexiquense de Cultura/Asociación Mexiquense de Cronistas Municipales, AC.

HSBC (2010). Información financiera al 30 de junio de 2010. Comunicado de Prensa. Informe Trimestral Segundo Trimestre 20I0. Recuperado el is de enero de 20I2, de https://www.hsbc.com.mx/I/PA_esf-ca-app-content/content/inicio/grupo_hsbc/ relacion_inversionistas/tabı_info_financiera/archivos/2_trimestre_ıo.pdf

Instituto Nacional de Estadística y Geografía e Informática [Inegi] (2010). Principales resultados por localidad (ITER). Censo de población y vivienda. Recuperado el 20 de marzo de 20II, de http://www.inegi.org.mx/sistemas/consulta_resultados/iter2oro.aspx

Korsbaek, L. (2009). El comunalismo: cambio de paradigma en la antropología mexicana a raíz de la globalización. Revista Argumentos, 22(59), I0I-I23. Recuperado el 5 de enero de 20I2, de http://redalyc.uaemex.mx /src/inicio/ArtPdfRed. jsp?iCve=595II4I2004 
Maldonado, B. (2002). Autonomía y comunalidad india. Enfoques y propuestas desde Oaxaca. Oaxaca: Centro INAH Oaxaca, Secretaría de Asuntos Indígenas del Gobierno de Oaxaca, Coalición de Maestros y Promotores Indígenas de Oaxaca AC, Centro de Encuentros y Diálogos Interculturales

Maldonado, B. (2003). Prólogo. Una forma comunal de ser en la sierra de Juárez. En J. Martínez, Comunalidad y desarrollo. México, D. F.: Cultura Indígena, Consejo Nacional para la Cultura y las Artes, Dirección General de Culturas Populares e Indígenas.

Martínez, J. (2003). Comunalidad y desarrollo. Cultura indígena. México, D. F.: Consejo Nacional para la Cultura y las Artes, Dirección General de Culturas Populares e Indígenas

Parkswatch (2002). México D. F.: Corredor Biológico Chichinantzin. Recuperado el 15 de febrero de 2013, de http://www.parkswatch.org/parkprofiles/pdf/chbc_spa.pdf Pérez-Ramírez, C., Zizumbo-Villarreal, L., Romero-Contreras, T., Cruz-Jiménez, G. y Madrigal-Uribe, D. (20II). El turismo como intervención e implicaciones para las comunidades rurales. Revista Gestión Turística, (16), 229-264.

Pérez-Ramírez, C., Zizumbo-Villarreal, L., Monterroso-Salvatierra, N., y

Madrigal-Uribe, D. (2012). Marco metodológico para el estudio del turismo rural. Perspectiva de análisis desde la comunalidad. Revista Estudios y Perspectivas en Turismo, 2I(2), 436-460. Recuperado el 3 de junio de 20I3, de http://www. estudiosenturismo.com.ar/PDF/V2I/No2/v2m2aio.pdf

Presidencia de la República (1936). Decreto que crea el Parque Nacional Lagunas de Zempoala, 27-II-I936. Recuperado el I2 de enero de 2009, de http://www.conanp. gob.mx/sig/decretos/parques/Zempoala.pdf Rendón, J. (2003). Comunalidad: modo de vida comunal entre los pueblos indios. México D. F.: Consejo Nacional para la Cultura y las Artes, Dirección General de Culturas Populares e Indígenas.

Rickards, J. y Piguerón, C. (2003). Financiamiento de programas para conservación de ecosistemas templados de montaña. En O. Sánchez, E. Vega, E. Peters y O. Monroy (Eds.), Conservación de ecosistemas templados de montaña en México (pp. 30I-315). México D. F.: Instituto Nacional de Ecología.

Romero, T. y Ávila, L. (200I). Eric R. Wolf: humanista y científico social del siglo XX. En T. Romero (Coord.), Historia de la ciencia en México: la antropología. Toluca: Universidad Autónoma del Estado de México.

World Bank (1972). Tourism. Sector Working Paper. Washington D.C.: Autor. Recuperado el 30 de octubre de 20I2, de http://go.worldbank.org/FDMUOLETSO 